\title{
The Existence and Uniqueness of a Class of Fractional Differential Equations
}

\author{
Zhanbing Bai, ${ }^{1}$ Sujing Sun, ${ }^{2}$ and YangQuan Chen ${ }^{3}$ \\ ${ }^{1}$ College of Mathematics and System Science, Shandong University of Science and Technology, Qingdao 266590, China \\ ${ }^{2}$ College of Information Science and Engineering, Shandong University of Science and Technology, Qingdao 266590, China \\ ${ }^{3}$ School of Engineering, University of California, Merced, CA 95343, USA
}

Correspondence should be addressed to Zhanbing Bai; zhanbingbai@163.com

Received 26 April 2014; Revised 30 May 2014; Accepted 31 May 2014; Published 17 June 2014

Academic Editor: Luigi Rodino

Copyright (C) 2014 Zhanbing Bai et al. This is an open access article distributed under the Creative Commons Attribution License, which permits unrestricted use, distribution, and reproduction in any medium, provided the original work is properly cited.

By using inequalities, fixed point theorems, and lower and upper solution method, the existence and uniqueness of a class of fractional initial value problems, $D_{0+}^{q} x(t)=f\left(t, x(t), D_{0+}^{q-1} x(t)\right), t \in(0, T), x(0)=0, D_{0+}^{q-1} x(0)=x_{0}$, are discussed, where $f \in C\left([0, T] \times \mathbb{R}^{2}, \mathbb{R}\right), D_{0+}^{q} x(t)$ is the standard Riemann-Liouville fractional derivative, $1<q<2$. Some mistakes in the literature are pointed out and some new inequalities and existence and uniqueness results are obtained.

\section{Introduction}

Once the models of fractional differential equation for the actual problem have been established, people immediately faced the problem of how to solve these models. In many cases, it is very difficult to obtain the exact solution of the fractional differential equation. So it requires researchers to find as many characteristics of the solution of the problem as possible. For example, does the equation have a solution? If there is one solution, is the solution unique? How can we compare the size of the solution? We noted that although there were many works with respect to fractional differential equations, which were shown in [1-10] and the references therein, the basic theory of the problem is still not perfect.

Al-Bassam [11] (1965) first considered the following Cauchy-type initial value problem (IVP):

$$
\begin{array}{cl}
D_{a+}^{\alpha} y(t)=f(t, y(t)), & (0<\alpha \leq 1), \\
\left.I_{a+}^{1-\alpha} y(t)\right|_{t=a}=b_{1}, & b_{1} \in \mathbb{R},
\end{array}
$$

in the space of continuous functions $C[a, b]$ provided that $f(t, y)$ is real-valued, continuous, and Lipschitzian in a domain $G \subset \mathbb{R} \times \mathbb{R}$ such that $\sup _{(t, y) \in G}|f(t, y)|=b_{0}<\infty$.
Applying the operator $I_{a+}^{\alpha}$ he reduced problem (1) to the Volterra nonlinear integral equation:

$$
y(t)=\frac{b_{1}(t-a)^{\alpha-1}}{\Gamma(\alpha)}+\frac{1}{\Gamma(\alpha)} \int_{a}^{t} \frac{f(s, y(s)) d s}{(t-s)^{1-\alpha}}, \quad(t>a) .
$$

By the use of the method of successive approximation he established the existence of the continuous solution of (2). He probably first indicated that the method of contracting mapping can be applied to prove the uniqueness of the solution of (2) and gave such a formal proof. However, from (2) one has $\lim _{t \rightarrow a+} y(t)=\infty$, so in space $C[a, b]$, the Cauchy-type problem (1) cannot be reduced to the integral equation (2) except that $b_{1}=0$.

Delbosco and Rodino [6] (1996) considered the nonlinear fractional differential equation

$$
D_{0+}^{\alpha} y(t)=f(t, y(t)), \quad 0 \leq t \leq 1 .
$$

Using Schauder's fixed point theorem to the integral operator in (2) with $a=b_{1}=0$, they proved that the equation considered has at least one continuous solution $y \in C[0, \delta]$ for a suitable $0 \leq \delta \leq 1$ provided that $t^{\sigma} f(t, y)$ is continuous 
on $[0,1] \times \mathbb{R}$ for some $\sigma(0 \leq \sigma<\alpha<1)$. Applying the contractive mapping method, they showed that if additionally

$$
|f(t, y)-f(t, z)| \leq \frac{M}{t^{\sigma}}|y-z|
$$

then (3) has a unique solution $y(t) \in C[0,1]$. Clearly, the solution satisfies $y(0)=0$. They also proved that if $f(t, y)=$ $f(y)$ is such that $f(0)=0$ and the Lipschitz condition holds, then the weighted Cauchy-type IVP

$$
\begin{aligned}
D_{0+}^{\alpha} y(t)= & f(y(t)), \quad \lim _{t \rightarrow 0} t^{1-\alpha} y(t)=c, \\
& (0<\alpha<1, c \in \mathbb{R})
\end{aligned}
$$

has a unique solution $y(t)$ such that $t^{1-\alpha} y(t) \in C[0, h]$ for any $h>0$.

In [12] (2008), Lakshmikantham and Vatsala considered the IVP for fractional differential equations given by

$$
D_{0+}^{\alpha} y(t)=f(t, y(t)), \quad y(0)=y_{0} .
$$

The basic theory for the IVP of fractional differential equations was discussed by employing the classical approach. The theory of inequalities, local existence, extremal solutions, comparison result, and global existence of solutions was considered. The idea of this paper is very interesting.

In [13] (2009), Zhang considered the existence and uniqueness of the solution of the following IVP for fractional differential equation:

$$
\begin{gathered}
D_{0+}^{\alpha} y(t)=f(t, y(t)), \quad 0 \leq t \leq T, \\
\left.t^{1-\alpha} y(t)\right|_{t=0}=y_{0},
\end{gathered}
$$

using the method of upper and lower solutions and its associated monotone iterative technique. However, the paper did not explain why the pointwise convergence can be used instead of the convergence with norm in the space $C_{1-\alpha}[0, T]$.

We refer the readers to monographs $[8,10]$ for other arguments about the fractional IVP. We noted that on one hand there are some confusions about the initial value of the solution in some of the above works. On the other hand there is no contribution about the basic theory for the following fractional differential equation IVP:

$$
\begin{gathered}
D_{0+}^{q} x(t)=f\left(t, x(t), D_{0+}^{q-1} x(t)\right), \quad t \in(0, T), \\
x(0)=0, \quad D_{0+}^{q-1} x(0)=x_{0},
\end{gathered}
$$

where $f \in C\left([0, T] \times \mathbb{R}^{2}, \mathbb{R}\right), D_{0+}^{q} x(t)$ is the standard Riemann-Liouville fractional derivative, $1<q<2$. This problem is very important in many models of physics phenomena $[7,9,10,14-16]$, so it is worth studying the parallel theory to the known theory of ordinary differential equations.

The rest of the paper is organized as follows. In Section 2, some related basic lemmas and definitions are given. Section 3 contains the uniqueness result by means of contracting mapping. The existence of the minimal and maximal solutions is given in Section 4 using lower and upper solution method.

\section{Preliminaries}

Lemma 1 (see $[3,8]$ ). For $y \in C^{n-1}(0, T) \cap L^{1}[0, T]$, the fractional differential equation

$$
D_{0+}^{\alpha} y(t)=0
$$

has a unique solution

$$
y(t)=\sum_{k=0}^{n-1} \frac{\left[D_{0+}^{\alpha-k-1} y(t)\right]_{t=0}}{\Gamma(\alpha-k)} t^{\alpha-k-1}
$$

Lemma 2 (see [10]). The following relation holds

$$
I_{0+}^{\alpha} I_{0+}^{\beta} \varphi=I_{0+}^{\alpha+\beta} \varphi,
$$

in the case of
(1) $\beta \geq 0, \alpha+\beta \geq 0, \varphi(t) \in L^{1}(0, T)$;
(2) $\beta \leq 0, \alpha \geq 0, \varphi(t) \in I_{0+}^{-\beta}\left(L^{1}(0, T)\right)$;
(3) $\alpha \leq 0, \alpha+\beta \leq 0, \varphi(t) \in I_{0+}^{-\alpha-\beta}\left(L^{1}(0, T)\right)$.

Lemma 3 (see [9]). Supposing that $n \in N, 0<\alpha \in \mathbb{R}$, then

$$
\frac{d^{n}}{d t^{n}}\left(D_{a+}^{\alpha} f(t)\right)=D_{a+}^{n+\alpha} f(t)
$$

(2)

$$
D_{a+}^{\alpha}\left(\frac{d^{n} f(t)}{d t^{n}}\right)=D_{a+}^{n+\alpha} f(t)-\sum_{j=0}^{n-1} \frac{f^{(j)}(a)(t-a)^{j-\alpha-n}}{\Gamma(1+j-\alpha-n)} .
$$

Lemma 4 (see [17]). Suppose that $E$ is an ordered Banach space, $x_{0}, y_{0} \in E, x_{0} \leq y_{0}, D=\left[x_{0}, y_{0}\right], A: D \rightarrow E$ is an increasing completely continuous operator, and $x_{0} \leq A x_{0}, y_{0} \geq$ $A y_{0}$. Then the operator $A$ has a minimal fixed point $x^{*}$ and $a$ maximal fixed point $y^{*}$. If one lets

$$
x_{n}=A x_{n-1}, \quad y_{n}=A y_{n-1}, \quad n=1,2,3, \ldots,
$$

then

$$
\begin{gathered}
x_{0} \leq x_{1} \leq x_{2} \leq \cdots \leq x_{n} \leq \cdots \leq y_{n} \leq \cdots \leq y_{2} \leq y_{1} \leq y_{0}, \\
x_{n} \longrightarrow x^{*}, \quad y_{n} \longrightarrow y^{*}
\end{gathered}
$$

By the use of the continuity of $f$ and Lemma 1, the IVP (8) is equivalent to the following Volterra integral equation [8]:

$$
\begin{aligned}
x(t)= & \frac{x_{0}}{\Gamma(q)} t^{q-1}+\frac{1}{\Gamma(q)} \\
& \times \int_{0}^{t}(t-s)^{q-1} f\left(s, x(s), D_{0+}^{q-1} x(s)\right) d s, \\
& 0 \leq t \leq T .
\end{aligned}
$$


Define the space

$$
C^{q}[0, T]:=\left\{x \mid x \in C[0, T], D_{0+}^{q-1} x \in C[0, T]\right\} .
$$

For $x \in C^{q}[0, T]$, define an operator $A: C^{q}[0, T] \rightarrow C[0, T]$ by

$$
\begin{aligned}
(A x)(t)= & \frac{x_{0}}{\Gamma(q)} t^{q-1}+\frac{1}{\Gamma(q)} \\
& \times \int_{0}^{t}(t-s)^{q-1} f\left(s, x(s), D_{0+}^{q-1} x(s)\right) d s .
\end{aligned}
$$

Then the fixed point of $A$ solves IVP (8) and vice versa.

Definition 5. A function $v(t) \in C^{q}[0, T]$ is called a lower solution of problem (8), if it satisfies

$$
\begin{gathered}
D_{0+}^{q} v(t) \leq f\left(t, v(t), D_{0+}^{q-1} v(t)\right), \quad t \in(0, T), \\
v(0)=0, \quad D_{0+}^{q-1} v(0) \leq x_{0}
\end{gathered}
$$

Definition 6. A function $w(t) \in C^{q}[0, T]$ is called an upper solution of problem (8), if it satisfies

$$
\begin{gathered}
D_{0+}^{q} w(t) \geq f\left(t, w(t), D_{0+}^{q-1} w(t)\right), \quad t \in(0, T), \\
w(0)=0, \quad D_{0+}^{q-1} w(0) \geq x_{0} .
\end{gathered}
$$

If one of the above inequalities is strict, then we call it as a strict lower (upper) solution.

Remark 7. Clearly, if functions $v, w$ are lower and upper solutions (or strict) of IVP (8), then there are $v \leq A v, w \geq A w$ (or the inequality is strict).

\section{The Uniqueness of the Solution}

Many methods can be applied to study the existence of solution. However, generally speaking, it is nothing more than two ways. One is based on the method of the approximate solution of exact solution to prove the existence of the solution, namely, classical successive approximation method. A. Cauchy, R. Lipschitz, G. Peano, and so forth used this method to solve the existence of some special types of differential equations. In 1893, C. Picard applied this method to study the general nonlinear differential equation and established the existence and uniqueness of solutions, named the Cauchy-Picard Theorem. This method itself also contains a structural method to obtain the exact solution and thus provides a way for the approximate solution. Another method is transforming the solution into the fixed point of some maps. Although the method cannot give the approximate solution, it is the abstraction and generalization of the former method and is simple to use. In this section, we will establish the uniqueness of the solution for fractional IVP (8) by the use of the second method.

Theorem 8. Assume that $f:[0, T] \times \mathbb{R}^{2} \rightarrow \mathbb{R}$ is continuous and Lipschitzian with respect to the second and the third variables; that is to say, there exist constants $L_{1}, L_{2}>0$ such that for all $x_{1}, y_{1}, x_{2}, y_{2} \in \mathbb{R}, t \in[0, T]$

$$
\left|f\left(t, x_{1}, y_{1}\right)-f\left(t, x_{2}, y_{2}\right)\right| \leq L_{1}\left|x_{1}-x_{2}\right|+L_{2}\left|y_{1}-y_{2}\right|
$$

Then the fractional IVP (8) has a unique solution $u \in C[0, T]$.

Proof. For $\varphi \in C^{q}[0, T]$, the norm is defined as

$$
\|\varphi\|=\max _{t \in[0, T]} e^{-\beta t}|\varphi(t)|+\max _{t \in[0, T]} e^{-\beta t}\left|D_{0+}^{q-1} \varphi(t)\right|,
$$

where $\beta$ is a positive constant such that

$$
\frac{\left(L_{1}+L_{2}\right)\left(\int_{0}^{T}(T-s)^{2 q-2} d s\right)^{1 / 2}}{\Gamma(q)(2 \beta)^{1 / 2}}<1 .
$$

Then $\left(C^{q}[0, T],\|\cdot\|\right)$ is a Banach space.

Clearly, the operator $A$ defined by (18) maps $C^{q}[0, T]$ to $C^{q}[0, T]$.

Now we prove that operator $A$ is a compressed map on $\left(C^{q}[0, T],\|\cdot\|\right)$. Let $y, z \in C^{q}[0, T]$; then, for $t \in[0, T]$,

$$
\begin{aligned}
& (A y)(t)-(A z)(t) \\
& =\frac{1}{\Gamma(q)} \int_{0}^{t}(t-s)^{q-1}\left[\begin{array}{r}
\left.f, y(s), D_{0+}^{q-1} y(s)\right) \\
\quad
\end{array}\right. \\
& \left.\quad-f\left(s, z(s), D_{0+}^{q-1} z(s)\right)\right] d s .
\end{aligned}
$$

Taking into account that the function $f$ is Lipschitzian, by the use of the Cauchy-Schwartz inequality, we have

$$
\begin{aligned}
& e^{-\beta t}|(A y-A z)(t)| \\
& \leq e^{-\beta t} \frac{1}{\Gamma(q)} \int_{0}^{t}(t-s)^{q-1} e^{\beta s} e^{-\beta s} \\
& \quad \times\left\{L_{1}|y(s)-z(s)|\right. \\
& \left.\quad+L_{2}\left|D_{0+}^{q-1} y(s)-D_{0+}^{q-1} z(s)\right|\right\} d s \\
& \leq \frac{\left(L_{1}+L_{2}\right) e^{-\beta t}}{\Gamma(q)} \int_{0}^{t}(t-s)^{q-1} e^{\beta s}\|y-z\| d s \\
& =\frac{\left(L_{1}+L_{2}\right) e^{-\beta t}\|y-z\| \int_{0}^{t}(t-s)^{q-1} e^{\beta s} d s}{\Gamma(q)}\left(\int_{0}^{t}(t-s)^{2 q-2} d s\right)^{1 / 2} \\
& \leq \frac{\left(L_{1}+L_{2}\right) e^{-\beta t}\|y-z\|\left(\int^{2}\right)}{\Gamma(q)}\left(\int_{0}^{t} e^{2 \beta s} d s\right)^{1 / 2}
\end{aligned}
$$




$$
\begin{aligned}
\leq & \frac{\left(L_{1}+L_{2}\right) e^{-\beta t}\|y-z\|}{\Gamma(q)}\left(\int_{0}^{t}(t-s)^{2 q-2} d s\right)^{1 / 2} \\
& \times \frac{e^{\beta t}}{(2 \beta)^{1 / 2}} \\
\leq & \frac{\left(L_{1}+L_{2}\right)\left(\int_{0}^{T}(T-s)^{2 q-2} d s\right)^{1 / 2}}{\Gamma(q)(2 \beta)^{1 / 2}}\|y-z\| .
\end{aligned}
$$

According to the definition of $\beta$, we know that $A$ : $C^{q}[0, T] \rightarrow C^{q}[0, T]$ is a compressed map. Banach fixed point theorem shows that there exists a unique $y \in C^{q}[0, T]$ such that $A y=y$; equivalently, $\operatorname{IVP}(8)$ has a unique solution $y \in C[0, T]$.

Remark 9. Similar to paper [6], we can permit function $f(t, x, y)$ to have some singularity on $t$.

Remark 10. The study about the following problem is meaningful:

$$
\begin{gathered}
D_{0+}^{q} x(t)=f\left(t, x(t), D_{0+}^{p} x(t)\right), \quad t \in(0, T), \\
x(0)=0, \quad D_{0+}^{q-1} x(0)=x_{0}
\end{gathered}
$$

where $f \in C\left([0, T] \times \mathbb{R}^{2}, \mathbb{R}\right), D_{0+}^{q} x(t)$ is the standard Riemann-Liouville fractional derivative, $1<p<q<2$.

\section{Some Inequalities and the Existence of the Solution}

Firstly, let us discuss the result about the strict inequalities for fractional IVP.

Theorem 11. Assume that the functions $v, w \in C^{q}[0, T]$ are lower and upper solutions of problem (8) and at least one of them is strict. For every $t \in[0, T], f(t, x, y)$ is nondecreasing about $x, y$. Then

$$
\begin{gathered}
D_{0+}^{q-1} v(t)<D_{0+}^{q-1} w(t), \quad 0<t \leq T \\
v(t)<w(t), \quad 0<t \leq T .
\end{gathered}
$$

Furthermore, the fractional IVP (8) has a minimal solution $x^{*}$ and a maximal solution $y^{*}$ such that

$$
x^{*}=\lim _{n \rightarrow \infty} A^{n} v, \quad y^{*}=\lim _{n \rightarrow \infty} A^{n} w .
$$

Proof. Without loss of generality, suppose that $D_{0+}^{q-1} w(0)>$ $x_{0}$. Let $y(t)=D_{0+}^{q-1} v(t)$. By the use of Lemma 3 and the definition of lower solution $v$, one has

$$
\begin{aligned}
y^{\prime}(t) & \leq f(t, v(t), y(t)), \quad t \in(0, T), \\
y(0) & \leq x_{0} .
\end{aligned}
$$

Integration from 0 to $t$ yields

$$
y(t) \leq x_{0}+\int_{0}^{t} f(s, v(s), y(s)) d s .
$$

Similarly, let $z(t)=D_{0+}^{q-1} w(t)$; we get

$$
z(t) \geq x_{0}+\int_{0}^{t} f(s, w(s), z(s)) d s .
$$

Suppose for contradiction that conclusion (27) is not true. Combining the fact that $D_{0+}^{q-1} v, D_{0+}^{q-1} w$ is continuous on $[0, T]$ and $y(0)=D_{0+}^{q-1} v(0) \leq x_{0}<D_{0+}^{q-1} w(0)=z(0)$, there exists $t_{1} \in(0, T]$ such that

$$
y\left(t_{1}\right)=z\left(t_{1}\right), \quad y(t)<z(t), \quad 0 \leq t<t_{1} .
$$

Taking into account that $D_{0+}^{q-1}(w(t)-v(t)):=h(t)>0$ for $0<t<t_{1}$ and $w(0)-v(0)=0$, by the use of the monotonicity of integral operator $I_{0+}^{q-1}$, one has

$$
v(t) \leq w(t), \quad 0 \leq t \leq t_{1} .
$$

With inequalities (33) and (34), $f$ is nondecreasing and above arguments give

$$
\begin{aligned}
z\left(t_{1}\right) & \geq x_{0}+\int_{0}^{t_{1}} f(s, w(s), z(s)) d s \\
& >x_{0}+\int_{0}^{t_{1}} f(s, v(s), y(s)) d s \geq y\left(t_{1}\right),
\end{aligned}
$$

which is a contradiction to (33). Thus, conclusion (27) holds. Furthermore, combining $v(0)=w(0)=0$ and the monotonicity of integral $I_{0+}^{q-1}$ yields that (28) also holds.

A standard proof can show that $A: C^{q}[0, T] \rightarrow C^{q}[0, T]$ is an increasing completely continuous operator. Setting $D:=$ $[v, w]$, by the use of Lemma 4 , the existence of $x^{*}, y^{*}$ is obtained. The proof is complete. ties.

The following conclusion is about the nonstrict inequali-

Theorem 12. Assume that the functions $v, w \in C^{q}[0, T]$ are lower and upper solutions of problem (8). If there exist two real numbers $L_{1}, L_{2}>0$ such that, for $t \in[0, T], x_{1} \geq x_{2}, y_{1} \geq y_{2}$, there holds

$$
\begin{aligned}
0 & \leq f\left(t, x_{1}, y_{1}\right)-f\left(t, x_{2}, y_{2}\right) \\
& \leq \frac{L_{1}}{t^{q}+t^{q-1}}\left(x_{1}-x_{2}\right)+\frac{L_{2}}{\Gamma(q)+\Gamma(q+1) t}\left(y_{1}-y_{2}\right),
\end{aligned}
$$

then $L_{1}+L_{2} \leq \Gamma(q+1)$ implies

$$
v(t) \leq w(t), \quad 0 \leq t \leq T .
$$

Furthermore, the fractional IVP (8) has a minimal solution $x^{*}$ and a maximal solution $y^{*}$ such that

$$
x^{*}=\lim _{n \rightarrow \infty} A^{n} v, \quad y^{*}=\lim _{n \rightarrow \infty} A^{n} w .
$$


Proof. Given $\epsilon>0$, let $w_{\epsilon}(t)=w(t)+\epsilon\left(t^{q}+t^{q-1}\right)$. Then, for $0 \leq t \leq T$

$$
D_{0+}^{q-1} w_{\epsilon}(t)=D_{0+}^{q-1} w(t)+\Gamma(q+1) \epsilon t+\Gamma(q) \epsilon,
$$

and specially,

$$
\begin{gathered}
D_{0+}^{q-1} w_{\epsilon}(0)=D_{0+}^{q-1} w(0)+\Gamma(q) \epsilon \\
w_{\epsilon}(0)=w(0), \quad w_{\epsilon}(t)>w(t), \quad \text { for } 0<t \leq T .
\end{gathered}
$$

From (39) and condition (36), combining the fact that

$$
\int_{0}^{t}(t-s)^{q-1} d s=t^{q} \int_{0}^{1}(1-\sigma)^{q-1} d \sigma=\frac{\Gamma(q)}{\Gamma(q+1)} t^{q}
$$

with the condition $L_{1}+L_{2}<\Gamma(q+1)$, one has

$$
\begin{aligned}
D_{0+}^{q} w_{\epsilon}(t)= & w(t)+\epsilon\left(t^{q}+t^{q-1}\right) \\
\geq & f\left(t, w(t), D_{0+}^{q-1} w(t)\right)+\Gamma(q+1) \epsilon \\
\geq & f\left(t, w_{\epsilon}(t), D_{0+}^{q-1} w_{\epsilon}(t)\right)+\Gamma(q+1) \epsilon \\
& -\frac{L_{1}\left(w_{\epsilon}(t)-w(t)\right)}{t^{q}+t^{q-1}} \\
& -\frac{L_{2}\left(D_{0+}^{q-1} w_{\epsilon}(t)-D_{0+}^{q-1} w(t)\right)}{\Gamma(q)+\Gamma(q+1) t} \\
\geq & f\left(t, w_{\epsilon}(t), D_{0+}^{q-1} w_{\epsilon}(t)\right)
\end{aligned}
$$

This inequality combined with $w_{\epsilon}(0)=0, D_{0+}^{q-1} w_{\epsilon}(0)=$ $D_{0+}^{q-1} w(0)+\Gamma(q) \epsilon>x_{0}$ shows that $w_{\epsilon}(t)$ is a strict upper solution of problem (8).

For $w_{\epsilon}(t), v(t)$, by the use of Theorem 11, we get $v(t)<$ $w_{\epsilon}(t), 0<t \leq T$. As $\epsilon>0$ is arbitrary, (37) holds.

The rest of the proof is just similar to Theorem 11 .

Remark 13. If we instead use condition (36) with the condition that there exists real number $L_{1}, L_{2}>0$ such that, for $t \in[0, T], x_{1} \geq x_{2}, y_{1} \geq y_{2}$,

$$
\begin{aligned}
0 & \leq f\left(t, x_{1}, y_{1}\right)-f\left(t, x_{2}, y_{2}\right) \\
& \leq L_{1}\left(x_{1}-x_{2}\right)+L_{2}\left(y_{1}-y_{2}\right),
\end{aligned}
$$

then Theorem 12 still holds as long as the condition

$$
\frac{L_{1} \Gamma(q)}{\Gamma(2 q)}\left[\frac{T^{q}}{2}+T^{q-1}\right]+\frac{L_{2} \Gamma(q)}{\Gamma(q+1)}\left[\frac{q T}{q+1}+1\right] \leq 1
$$

is used instead of the condition $\Gamma(q+1) \geq L_{1}+L_{2}$.

\section{Conflict of Interests}

The authors declare that there is no conflict of interests regarding the publication of this paper.

\section{Acknowledgments}

This work is supported by NNSF $(61174078,61201431)$, SDNSF (ZR2010AM035), a project of Shandong Higher Educational Science and Technology program (J11LA07), the Taishan Scholar project, Research Award Fund for Outstanding Young Scientists of Shandong (BS2012SF022), and SDUST Research Fund (2011KYTD105). The authors thank the referee for his/her valuable comments and constructive suggestions.

\section{References}

[1] R. P. Agarwal, D. O'Regan, and S. Staněk, "Positive solutions for mixed problems of singular fractional differential equations," Mathematische Nachrichten, vol. 285, no. 1, pp. 27-41, 2012.

[2] B. Ahmad and J. J. Nieto, "Existence results for nonlinear boundary value problems of fractional integrodifferential equations with integral boundary conditions," Boundary Value Problems, vol. 2009, Article ID 708576, 11 pages, 2009.

[3] Z. Bai and H. Lü, "Positive solutions for boundary value problem of nonlinear fractional differential equation," Journal of Mathematical Analysis and Applications, vol. 311, no. 2, pp. 495-505, 2005.

[4] Z. Bai, "On positive solutions of a nonlocal fractional boundary value problem," Nonlinear Analysis: Theory, Methods \& Applications, vol. 72, no. 2, pp. 916-924, 2010.

[5] M. Benchohra, S. Hamani, and S. K. Ntouyas, "Boundary value problems for differential equations with fractional order and nonlocal conditions," Nonlinear Analysis: Theory, Methods \& Applications, vol. 71, no. 7-8, pp. 2391-2396, 2009.

[6] D. Delbosco and L. Rodino, "Existence and uniqueness for a nonlinear fractional differential equation," Journal of Mathematical Analysis and Applications, vol. 204, no. 2, pp. 609-625, 1996.

[7] V. Kiryakova, Generalized Fractional Calculus and Applications, vol. 301 of Pitman Research Notes in Mathematics Series, Longman, Harlow, UK, 1994.

[8] A. A. Kilbas, H. M. Srivastava, and J. J. Trujillo, Theory and Applications of Fractional Differential Equations, vol. 204, Elsevier Science B.V., Amsterdam, The Netherlands, 2006.

[9] I. Podlubny, Fractional Differential Equations, vol. 198, Academic Press, New York, NY, USA, 1999.

[10] S. G. Samko, A. A. Kilbas, and O. I. Marichev, Fractional Integrals and Derivatives, Theory and Applications, Gordon and Breach, Amsterdam, The Netherlands, 1993.

[11] M. A. Al-Bassam, "Some existence theorems on differential equations of generalized order," Journal für die Reine und Angewandte Mathematik, vol. 218, pp. 70-78, 1965.

[12] V. Lakshmikantham and A. S. Vatsala, "Basic theory of fractional differential equations," Nonlinear Analysis: Theory, Methods \& Applications, vol. 69, no. 8, pp. 2677-2682, 2008.

[13] S. Zhang, "Monotone iterative method for initial value problem involving Riemann-Liouville fractional derivatives," Nonlinear Analysis: Theory, Methods \& Applications, vol. 71, no. 5-6, pp. 2087-2093, 2009.

[14] K. Diethelm and N. J. Ford, "Analysis of fractional differential equations," Journal Fractional Calculus, vol. 7, pp. 89-100, 1995.

[15] K. Diethelm and N. J. Ford, "Analysis of fractional differential equations," Journal of Mathematical Analysis and Applications, vol. 265, no. 2, pp. 229-248, 2002. 
[16] C. A. Monje, Y. Chen, B. M. Vinagre, D. Xue, and V. Feliu, Fractional-Order Systems and Controls: Fundamentals and Applications, Springer, London, UK, 2010.

[17] D. J. Guo, J. X. Sun, and Z. L. Liu, Functional Methods in Nonlinear Ordinary Differential Equations, Shandong University of Science and Technology, Jinan, China, 1995, (Chinese). 


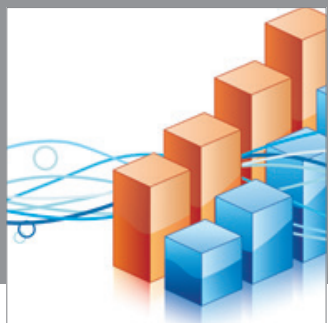

Advances in

Operations Research

mansans

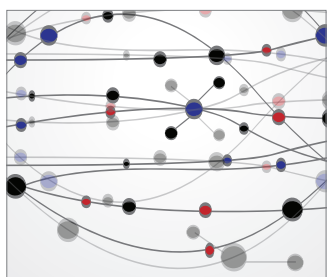

The Scientific World Journal
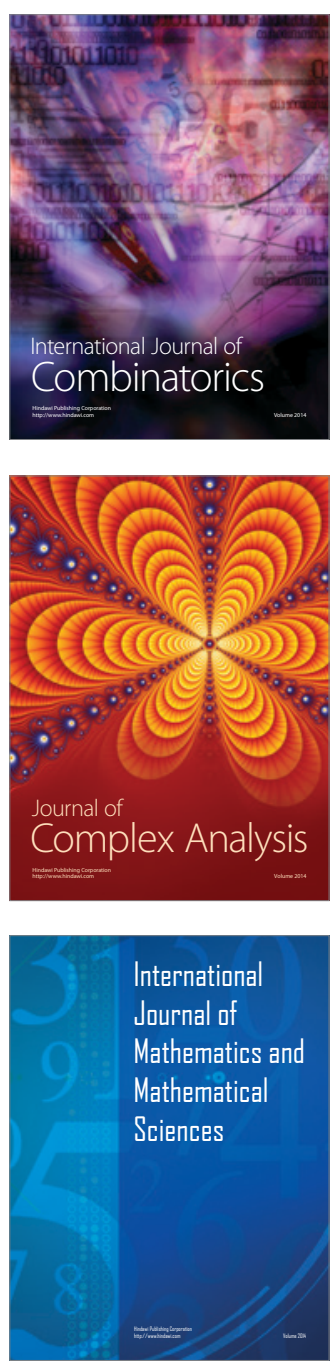
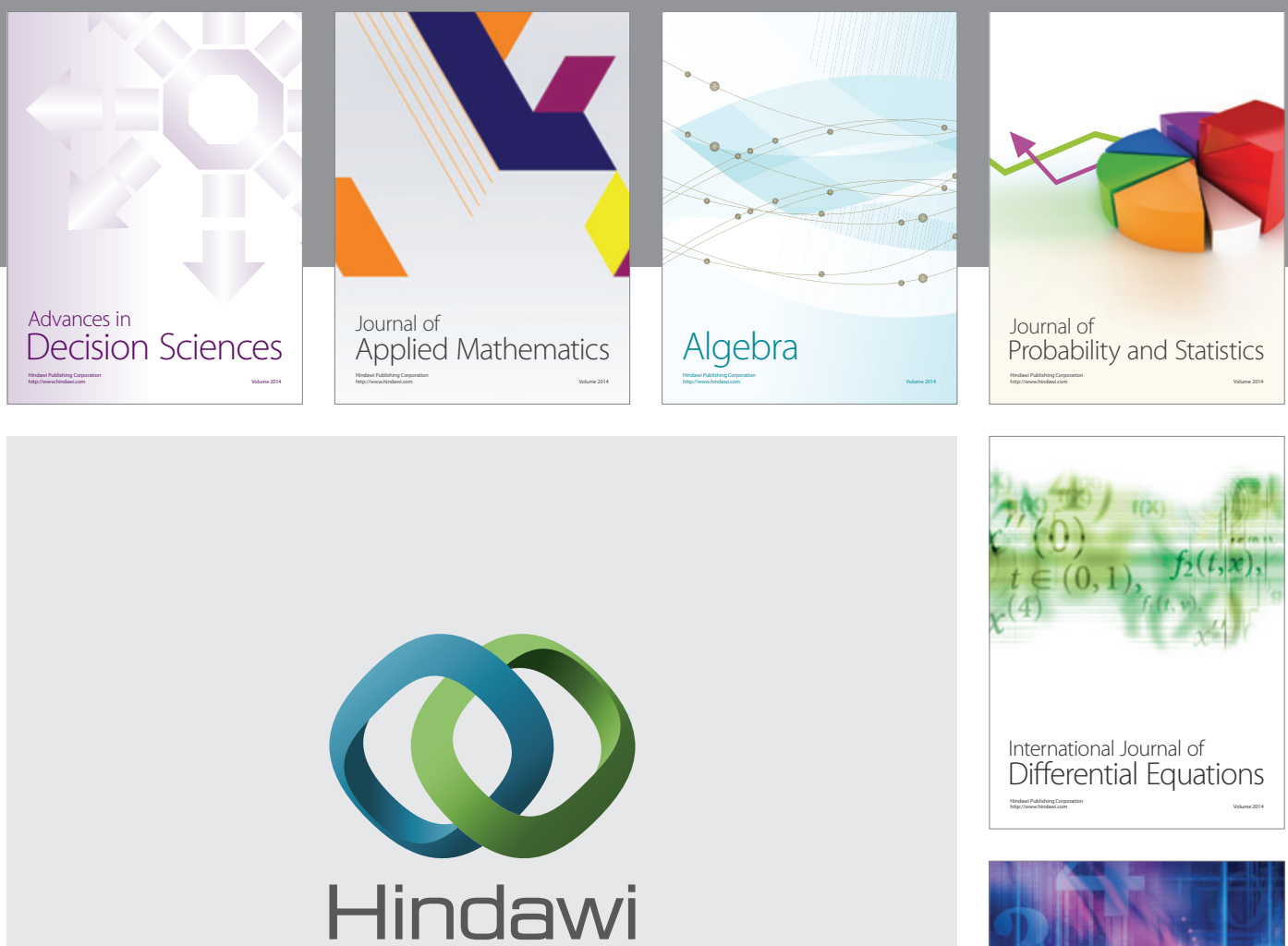

Submit your manuscripts at http://www.hindawi.com
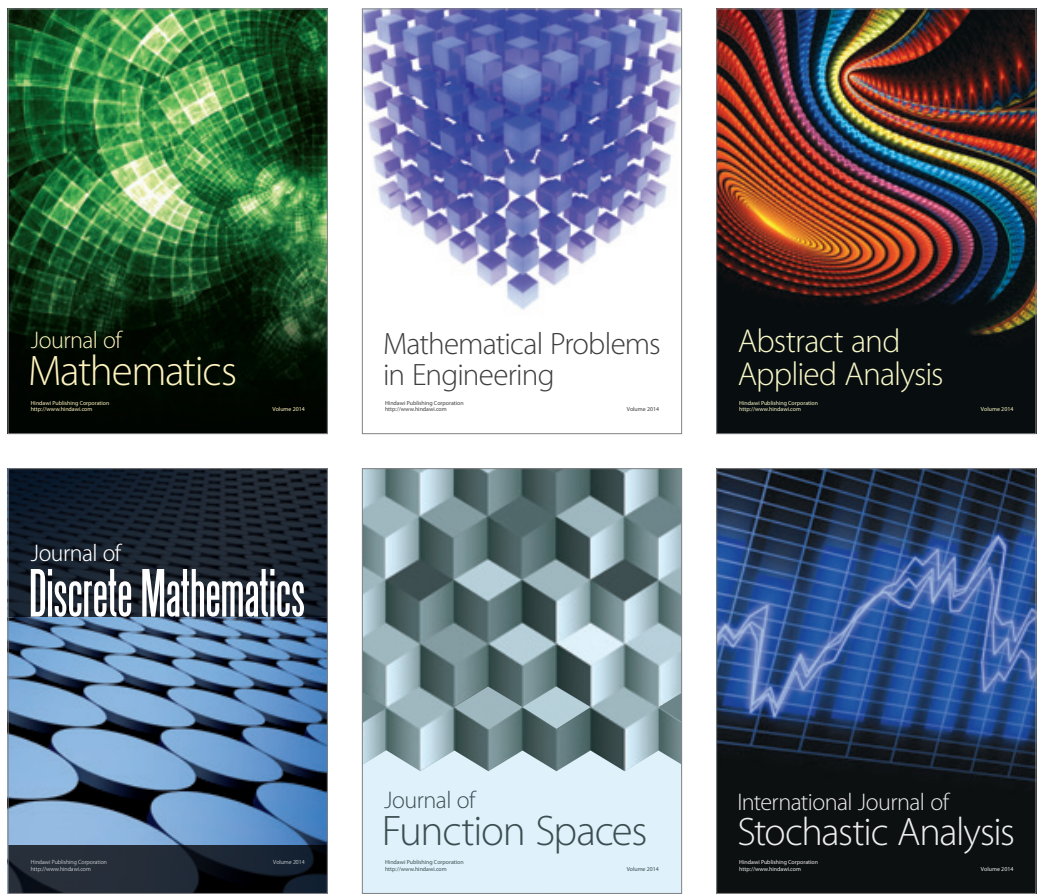

Journal of

Function Spaces

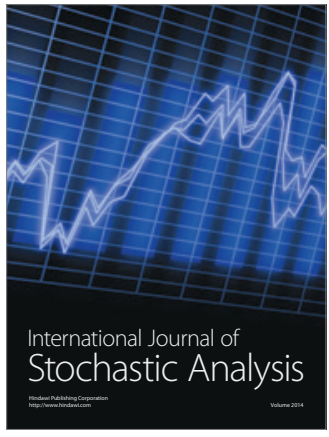

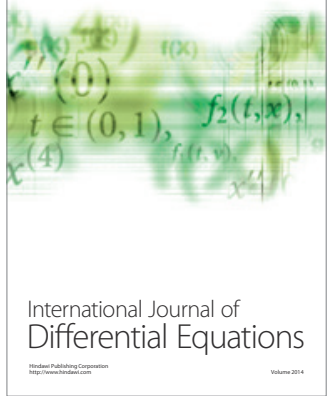
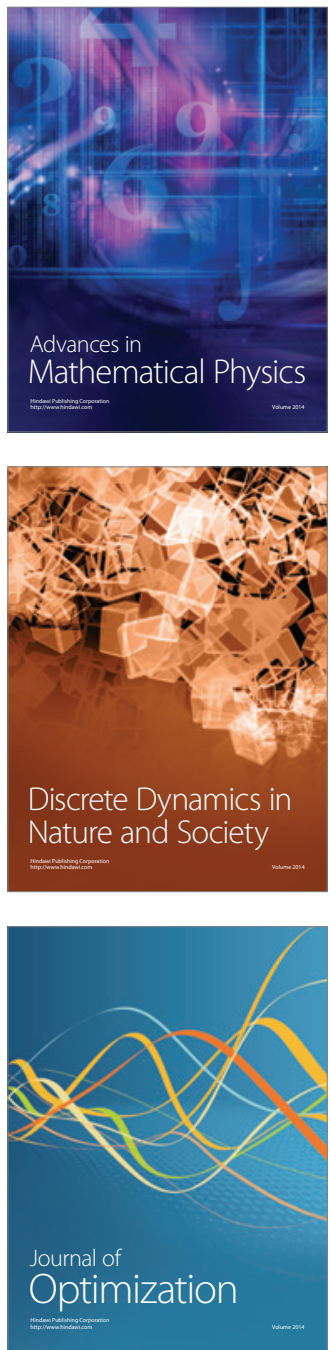